\title{
Design of singlet fission chromophores with cyclic (alkyl)(amino) carbene building blocks.
}

\author{
Achini Japahuge ${ }^{1}$, Seunghoon $\mathrm{Lee}^{2}$, Cheol Ho Choi ${ }^{3}$, and Tao Zeng ${ }^{1 \mathrm{a}}$ \\ ${ }^{1}$ Department of Chemistry, Carleton University, Ottawa, Ontario K1S5B6, Canada \\ 2 Department of Chemistry, Seoul National University, Seoul 151-747, South Korea \\ 3 Department of Chemistry, Kyungpook National University, Daegu 702-701, South Korea
}

\begin{abstract}
We use MRSF-TDDFT and NEVPT2 methods to design singlet fission chromophores with the building blocks of cyclic (alkyl)(amino)carbenes (CAACs). CAAC dimers with $\mathrm{C}_{2}, \mathrm{C}_{4}$, and $p$ phenylene spacers are considered. The substitutions with trifluoromethyls and fluorine atoms at the $\alpha \mathrm{C}$ position are investigated. The electronegative substituents enhance the $\pi$ accepting capability of the $\alpha \mathrm{C}$, while maintaining it as a quaternary $\mathrm{C}$ atom. The phenylene-connected dimers with the two substitutions are identified as promising candidates for singlet fission chromophores. The cylindrically symmetric $\mathrm{C}_{2}$ and $\mathrm{C}_{4}$ spacers allow for substantial structural reorganizations in the $S_{0}$-to- $S_{1}$ and $S_{0}$-to- $T_{1}$ excitations. Although the two substituted dimers with the $\mathrm{C}_{4}$ spacer satisfy (or very close to satisfy) the primary thermodynamics criterion for singlet fission, the significant structural reorganizations result in high barriers so that the fission is kinetically unfavorable.
\end{abstract}

\footnotetext{
a toby.zeng@carleton.ca
} 


\section{INTRODUCTION}

In an organic photovoltaic (OPV) device, the incidence of a photon on the chromophore layer creates one singlet exciton, which may diffuse to the chromophore/acceptor interface and undergo charge separation to create a pair of free charge carriers. However, the singlet excitons are usually short-lived due to their radiative and radiationless decays to ground state. These detrimental channels lower the photoelectric conversion efficiency of OPV devices. If the chromophore molecule satisfies certain energy relations between its excited states, the singlet exciton may fission into two triplet excitons. This process, called singlet fission $(\mathrm{SF}),{ }^{1,2}$ results in twice more excitons, and these triplet excitons do not decay to ground state rapidly due to the spin-conservation selection rule of the two decay channels. With the larger number and the longer life of the so generated excitons, SF has the potential to enhance the photoelectric conversion efficiency of a single-junction photovoltaic device to exceed the $\sim 30 \%$ Shockley-Queisser limit $^{3}$ and reach $\sim 45 \%{ }^{4}$

The two energy criteria for SF chromophores are a $^{1,2,5,6}$

$$
\begin{aligned}
& E\left(S_{1}\right) \geq 2 E\left(T_{1}\right) ; \\
& E\left(T_{2}\right) \geq 2 E\left(T_{1}\right) .
\end{aligned}
$$

$S$ and $T$ are used to label states in singlet and triplet spin manifolds. The subscripts 1 and 2 indicate the first and second excited states in the respective spin manifolds. The first inequality guarantees that the fission of one $S_{1}$ exciton to two $T_{1}$ excitons is exoergic and thermodynamically favorable. The second inequality ensures that the fusion of two SFgenerated $T_{1}$ excitons to one $T_{2}$ exciton is endoergic and thermodynamically unfavorable. In terms of spin angular momentum addition, two $T_{1}$ excitons can also fusion to one $Q_{1}$ exciton (the lowest quintet excited state). However, $E\left(Q_{1}\right)$ is usually greater than $2 E\left(T_{1}\right)$ and the path to the quintet manifold is hindered.

One obstacle for the application of the SF phenomenon in photovoltaics is that only a small number of molecules meet the two criteria: e.g., tetracene, ${ }^{7}$ pentacene, ${ }^{8-10}$ some of their derivatives, ${ }^{11-21}$ perylenediimide, ${ }^{22-24}$ and some polyenes. ${ }^{25-27}$ Especially, research of SF is "overwhelmingly limited" to tetracene and pentacene. ${ }^{28}$ There is thus a strong motivation to design more SF chromophores that meet the two criteria. It has been clarified by a series of theoretical studies that the possession of non-negligible diradical character and 
the lack of tetraradical character are the underlying reasons for a molecule to satisfy the two inequalities, respectively. ${ }^{29-33}$ Stable molecules, either isolated in reality or optimized in silico, rarely possess significant tetraradical character. Therefore, it would be relatively easier to satisfy Eq. 2 than Eq. 1. Eq. 1 is thus the primary criterion for SF chromophores. One approach to design SF chromophores is to enhance diradical character of a typical closed-shell molecule by introducing heteroatoms and substitutents. 1,3-Diphenylisobenzofuran, the first designed chromophore that exhibits SF behavior, was proposed following this strategy. ${ }^{34-36}$

This understanding stimulated a series of theoretical studies in designing SF chromophores, e.g., by introducing captodative effect ${ }^{37-42}$ and by introducing Si atom. ${ }^{43}$ These modifications shall be applied to structures that are by themselves not too low in diradical character. Otherwise, the enhancement in diradical character may not be enough to satisfy Eq. 1. Recently, attentions have been drawn to structures with cyclic (alkyl)(amino)carbenes (CAACs) as building blocks. ${ }^{44}$ CAACs are persistent singlet carbenes. ${ }^{45}$ Last year, a modular approach for connecting CAACs by spacers to form stable Kekulé diradicaloids was reported by the Bertrand group. ${ }^{46}$ This approach allows to tune the diradical character of the CAAC-based diradicaloids through modifying the spacer, especially its length and aromaticity. Therefore, this approach opens the doorway to design CAAC-based SF chromophores. Immediately following this work, Messelberger et al. performed a computational study of a series of CAAC-spacer-CAAC dimers and identified several candidates as SF chromophores. ${ }^{44}$ It is noteworthy that while CAACs are usually used as stabilizers for radical and diradical moieties that are attached to them, ${ }^{47-51}$ they are radical centers in those SF chromophore candidates.

Inspired by these latest advances in the field of SF, we in this work perform quantum chemistry calculations to study the possibility of designing CAAC-spacer-CAAC SF chromophores through fluorination and trifluoromethylation at the $\alpha \mathrm{C}$. The ideas of fluorination and trifluoromethylation originate from the captodative effect: we can stabilize a radical center by simultaneously attaching a donor and an acceptor to it. ${ }^{52,53}$ By connecting a CAAC unit to a spacer, one electron of the carbene's $\sigma$ lone pair participates in forming a $\sigma$ bond with the spacer. The leftover unpaired electron occupies the $\pi$ orbital of the carbene, making the carbene a $\pi$ radical. The $\mathrm{N}$ atom adjacent to the carbene $\mathrm{C}$ exerts a donating effect to the singly occupied carbene $\pi$ orbital. If the $\alpha \mathrm{C}$ can serve as a $\pi$ acceptor, the CAAC $\pi$ radical unit will be stabilized by the captodative effect of the $\alpha \mathrm{C}$ and the $\mathrm{N}$, and the 
diradical character of the CAAC-spacer-CAAC structure will be enhanced. The structure is then more likely to satisfy Eq. 1. As we show below, fluorination and trifluoromethylation at the $\alpha \mathrm{C}$ does enhance its $\pi$ accepting capability. Only homodimers are considered in this work, since heterodimers are likely to feature more or less ionic character in their ground state, which is exclusive with diradical character.

In Section II, we briefly overview the computational methods used in this work. The computational results are presented and discussed in Section III. Seciton IV concludes the paper.

\section{COMPUTATIONAL METHODS}

Recently, some of us (SL and CHC) developed the mixed-reference spip-flip timedependent density functional theory (MRSF-TDDFT) formalism, ${ }^{54}$ and its analytic energy gradient. ${ }^{55}$ The new theory is an improved SF-TDDFT, which can treat both dynamic and non-dynamic correlations in a balanced way. This theory overcomes the notorious deficiencies of the conventional SF-TDDFT known as the spin-contamination problem. It is shown that MRSF-TDDFT gives improved results than those of SF-TDDFT in calculating excitation energies. ${ }^{55}$ In the MRSF-TDDFT, the lowest triplet state obtained at the restricted open-shell DFT level is used as the reference state. The singlet and triplet response states of the same molecular structure are obtained by the linear response, i.e., one-electron transitions, from the same reference state, which is similar to configuration interaction singles. Although the orbitals of the reference states are optimized for the lowest-triplet reference state, the singlet and triplet response states are described by orbital rotations an occupied molecular orbitals to virtual orbitals of reference state. Therefore, the MRSF-TDDFT method does not bias towards the triplet response state that resembles the reference state. Geometry optimizations for all studied structures, in their ground and excited states in both singlet and triplet spin manifolds, are performed using the MRSF-TDDFT method. The method is also used for all hessian calculations.

All reported optimized dimer structures are of $C_{2}$ symmetry, unless further specified. Coordinates of all structures are given in Section S.II in the Supporting Information. Optimizations with $C_{i}$ symmetry for several representative molecules give similar energies and structural parameters. For instance, the $(\mathrm{CAAC})_{2}-\mathrm{C}_{2}$ dimer structures with $C_{2}$ and $C_{i}$ 
symmetries have identical bond lengths at their cumulene fragments (see Figure 2(a) for the bond lengths). Their calculated vertical $E\left(S_{1}\right)$ s and $E\left(S_{2}\right)$ s differ by only 0.002 and $0.006 \mathrm{eV}$, respectively. We hence focus our discussion on the $C_{2}$ structures. The BHHLYP functional ${ }^{56,57}$ and the Def2-SVP basis $\operatorname{set}^{58}$ are used in the optimization and hessian calculations, which are performed using a development version of the GAMESS-US program package. ${ }^{59,60}$ The hessian calculations confirm that the optimized structures are true minima on potential energy surfaces. We optimize water dimer structure using the Def2-SVP and Def2-TZVPP basis sets and the BHHLYP functional. The resultant $\mathrm{O} \cdots \mathrm{O}$ distances are 2.86 and $2.90 \AA$, respectively. The $0.04 \AA$ small difference corroborates the competence of the economical Def2-SVP basis set in optimizing structures with hydrogen bonding interaction. Hydrogen bonding interaction plays an important role in determining the structures of the dimers that are considered in this study (vide infra). All our reported energies contain ZPE corrections, unless further specified. The rapid geometry relaxation associated with photoexcitation competes with SF. ${ }^{1}$ It is hence more reasonable to use ZPE-corrected minimum-to-minimum (i.e., adiabatic) excitation energies to judge whether a molecule satisfies Eqs. 1 and 2. As shown below, structural reorganizations induced by excitation can be significant. It is well known that such excitation-induced reorganizations play an important role in energy transfer and charge transfer of organic semiconductors. ${ }^{61-63}$ In general, significant reorganizations lead to higher barriers and lower rates of the transfers.

All reported excitation energies are calculated using the second order n-electron valence state perturbation theory (NEVPT2) method ${ }^{64-66}$ implemented in the ORCA program package, ${ }^{67}$ with the Def2-TZVPP basis set. ${ }^{58}$ Full $\pi$ valence active spaces are used in the NEVPT2 calculations. Please note that the exclusion of the $\mathrm{N}$ lone pair orbitals from the active space overestimates the vertical $E\left(S_{1}\right)$ of the $(\mathrm{CAAC})_{2}-\mathrm{C}_{2}$ dimer by $0.32 \mathrm{eV}$ in a test calculation. It is thus necessary to include them, i.e., to use the full valence $\pi$ active space. The NEVPT2 wave functions are consistent with those obtained in the MRSF-TDDFT calculations. Therefore, the geometry optimizations and excitation energy calculations are performed for the same set of states.

In the NEVPT2 calculations, states that are relevant in an excitation are averaged equally. For instance, to calculate adiabatic $E\left(S_{1}\right)$ of a molecule, NEVPT2 calculations weighting $S_{0}$ and $S_{1}$ equally are carried out at $S_{0^{-}}$and $S_{1}$-optimized structures. The difference between $E\left(S_{1}\right)$ at $S_{1}$ structure and $E\left(S_{0}\right)$ at $S_{0}$ structure (abbreviated as $E\left(S_{0}\right) @ S_{0} \mathrm{~g}$ and 
$\left.E\left(S_{1}\right) @ S_{1} \mathrm{~g}\right)$, offset by the ZPEs of the respective structures, gives the adiabatic $E\left(S_{1}\right)$. We gradually include more states in the average to ensure that proper states have been captured by the minimum average scheme. If not, then more states are included until convergences of the energies and wave functions of the interesting states are attained. To calculate an adiabatic excitation energy, we always average the same number of states in the NEVPT2 calculations at the two optimized structures.

The CAAC model adopted in this work is shown in Figure 1(a), with all substituents on the 5-membered ring being methyls. The two $\mathrm{R}$ groups at the $\alpha \mathrm{C}$ atom are to be replaced by $\mathrm{F}$ atoms and trifluoromethyls to tune the electron-accepting capacity of the $\alpha \mathrm{C}$. The resultant CAAC structures are accordingly labelled as $\mathrm{CAAC}(\mathrm{F})$ and $\mathrm{CAAC}(\mathrm{TFM})$.

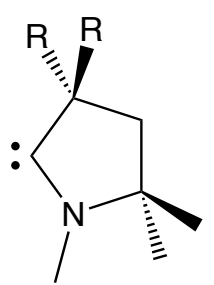

(a)

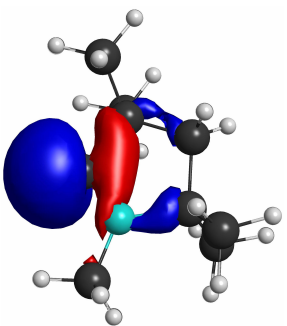

(b)

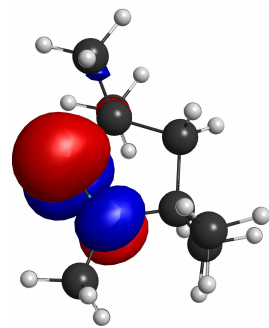

(c)

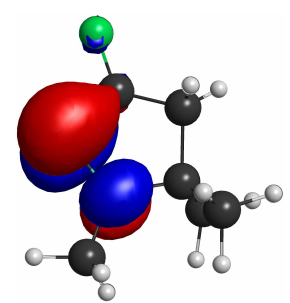

(d)

FIG. 1. (a) CAAC model used in this work; (b) and (c) the $\sigma$ HOMO and $\pi$ LUMO of CAAC in its singlet ground state; (d) the $\pi$ LUMO of CAAC(F).

\section{RESULTS AND DISCUSSION}

\section{A. The Fluorination and Trifluoromethylation}

The HOMO and LUMO of CAAC in its $S_{0}$ state are shown in Figure $1(\mathrm{~b})$ and (c). With the $\mathrm{N}$ as a $\pi$ donor, the $\pi$ LUMO features antibonding character between the $\mathrm{N}$ atom and the carbene $\mathrm{C}$ atom, and its energy is raised. With the lone pair electrons occupying the $\sigma \mathrm{HOMO}, \mathrm{CAAC}$ is a typical $\sigma$ donating N-heterocyclic carbene (NHC). In the CAAC $\pi$ LUMO, there is almost no bonding or antibonding interaction between the carbene $\mathrm{C}$ atom and the $\alpha \mathrm{C}$ atom. Quantitatively, the $\pi$ LUMO contributes -0.001 Mayer bond order ${ }^{68}$ between the two atoms. Once we replace the two methyls attached to the $\alpha \mathrm{C}$ by $\mathrm{F}$ atoms, the $\mathrm{C}-\mathrm{F} \sigma^{*}$ antibonding orbitals have lower energy and are more localized on the $\alpha \mathrm{C}$. The 
$\sigma^{*}$ orbitals will then become more effective in overlapping with the $p_{\pi} \mathrm{AO}$ on the carbene C. For comparison, the $\pi$ LUMO contributes 0.091 Mayer bond order between the two C atoms in $\mathrm{CAAC}(\mathrm{F})$. This typical through-bond bonding interaction ${ }^{69}$ lowers the energy of the $\pi$ LUMO and stabilizes the radical with the unpaired electron in the $\pi$ orbital.

The comparison of Figure 1(c) and (d) clearly shows that with the more electronegative $\mathrm{F}$ atoms, the $\pi$ LUMO features some bonding interaction between the carbene $\mathrm{C}$ and the $\alpha \mathrm{C}$. As a reflection of the stabilization of the $\pi \mathrm{LUMO}$, the vertical $E\left(T_{1}\right)$ of CAAC(F) is calculated to be lower than that of CAAC by $0.11 \mathrm{eV}(2.61 \mathrm{vs} .2 .72 \mathrm{eV})$, and the $T_{1}$ state has one electron in each of the $\sigma$ and the $\pi$ orbital. CAAC(TFM) is in between the other two structures. The $\pi$ LUMO contributes 0.012 Mayer bond order between the carbene $\mathrm{C}$ atom and the $\alpha \mathrm{C}$ atom in CAAC(TFM), and the vertical $E\left(T_{1}\right)$ is $2.67 \mathrm{eV}$. The BHHLYP HOMO-LUMO gaps are 4.57, 4.55, and $4.47 \mathrm{eV}$ for CAAC, CAAC(TFM), and CAAC(F). The gap decrease is consistent with the decrease of the vertical $E\left(T_{1}\right)$ and the increase of the Mayer $\pi$ bond order between the carbene $\mathrm{C}$ atom and the $\alpha \mathrm{C}$ atom. We are aware that according to the $\sigma$ electron donor-acceptor indicator proposed by Ozimiński et al., $\mathrm{CF}_{3}$ is less electronegative than $\mathrm{CH}_{3} .{ }^{70}$ With no intention to question the validity of this useful indicator, we here just present a trend that is in line with the chemical intuition: the presence of the $\mathrm{F}$ atoms in $\mathrm{CF}_{3}$ makes it more electronegative than $\mathrm{CH}_{3}$, and the $\alpha \mathrm{C}$ atom with the two $\mathrm{CF}_{3}$ groups exhibits a stronger $\pi$ accepting capability. The inconsistence is partially attributed to the complexity in defining electronegativity,${ }^{71}$ especially electronegativity of functional groups. ${ }^{72}$

There are other ways to stabilize the $\pi$ LUMO of a carbene. One straightforward way is to put a $\pi$ acceptor, e.g., a tertiary $\mathrm{B}$ atom, beside the carbene $\mathrm{C}$. We here adopt the fluorination and trifluoromethylation strategies, with the purpose to minimize the modification of the CAAC structure. It is of critical importance to keep a quaternary $\mathrm{C}$ in the $\alpha$ position, which provides steric protection and enhances the stability of CAACs. ${ }^{45}$ The results presented in this work show that it is possible to stabilize the $\pi$ LUMO while maintaining a quaternary $\alpha \mathrm{C}$. 


\section{B. CAAC Dimers with a $\mathrm{C}_{2}$ Spacer}

We first examine the dimers of the three types of CAAC units connected by a $\mathrm{C}_{2}$ spacer. The dimers are called $(\mathrm{CAAC})_{2}-\mathrm{C}_{2},(\mathrm{CAAC}(\mathrm{TFM}))_{2}-\mathrm{C}_{2}$, and $(\mathrm{CAAC}(\mathrm{F}))_{2}-\mathrm{C}_{2}$, respectively. These structures are of special interest as the $(\mathrm{CAAC})_{2}-\mathrm{C}_{2}$ cumulene has been isolated in good yield. ${ }^{73,74}$ It is thus desirable to investigate the (F) and (TFM) analogues with the same skeleton.

The optimized structures of the $S_{0}, T_{1}$, and $S_{1}$ states of $(\mathrm{CAAC})_{2}-\mathrm{C}_{2}$ are shown in Figure 2(a)-(c) and the calculated results are summarized in Table I. The $172^{\circ}$ dihedral angle $d_{\mathrm{NCCN}}$ indicates a cumulene resonance structure for this molecule. The occupancy of the lowest unoccupied natural orbital $\left(n_{\mathrm{LUNO}}\right)$ is 0.13 , which indicates the diradical character of this molecule. ${ }^{75}$ The $n_{\text {LUNO }}$ mainly arises from the configuration with the HOMO-to-LUMO two-electron excitation configuration. The orbitals are shown in Figure 2(d) and (e). This

excitation corresponds to polarizing the distributions of the two electrons in HOMO, so that when one electron occupies the $\pi$ orbital of one carbene, the other occupies the $\pi$ orbital of the other carbene. This suggests an increase of diradical character with the $\pi$ radical centers on the carbene $\mathrm{C}$ atoms. Meanwhile, the out-of-plane $\pi$ bonding orbital between the two central $\mathrm{C}$ atoms is strengthened due to the corresponding (anti)bonding character in the LUMO (HOMO). Therefore, the $S_{0}$ state adopts the additional diradical resonance structure shown in Figure 2(h). In consistence with the diradical resonance structure, the central CC bond of the cumulene moiety is shorter than the other two (1.26 vs. $1.33 \AA$ ). Please note that these calculated bond lengths are in excellent agreement with the experimental values of 1.27 and $1.33 \AA .73$ The XRD-determined bond lengths are for the single crystal of $(\mathrm{CAAC})_{2}-\mathrm{C}_{2}$ with diisopropylphenyls (Dipp) attached to the $\mathrm{N}$ atoms. The agreement reflects the accuracy of the MRSF-TDDFT structure optimization and the appropriateness of replacing the Dipp groups by methyls.

The $T_{1}$ state corresponds to the HOMO-to-LUMO single electron excitation, resulting in the pure diradical resonance structure shown in Figure 2(i). The resonance structure is consistent with the $T_{1}$ geometrical structure: the $1.24 \AA$ short $\mathrm{CC}$ triple bond in the center and the almost perpendicular dihedral angle made by the two CAAC fragments $\left(d_{\mathrm{NCCN}}=97^{\circ}\right)$. The ZPE-corrected vertical and adiabatic $E\left(T_{1}\right) \mathrm{s}$ are calculated to be 2.53 and $1.52 \mathrm{eV}$, respectively. The $1.01 \mathrm{eV}$ large reorganization energy is consistent with the 
TABLE I. Calculated results for the three $\mathrm{C}_{2}$-connected dimers. All energies are given in eV.

\begin{tabular}{cccc}
\hline & CAAC & $($ TFM $)$ & $(\mathrm{F})$ \\
\hline Adiabatic $E\left(S_{1}\right)$ & 2.15 & 2.47 & 2.33 \\
Vertical $E\left(S_{1}\right)(f)$ & $3.71(0.002)$ & $3.66(0.003)$ & $3.71(0.004)$ \\
Adiabatic $E\left(S_{2}\right)^{a}$ & 3.50 & 3.36 & 3.30 \\
Vertical $E\left(S_{2}\right)(f)$ & $3.71(0.76)$ & $3.55(0.75)$ & $3.53^{b}(0.75)$ \\
Adiabatic $E\left(T_{1}\right)$ & 1.52 & 1.43 & 1.29 \\
Vertical $E\left(T_{1}\right)$ & 2.53 & 2.33 & 2.19 \\
$n_{\mathrm{LUNO}}^{c}$ & 0.13 & 0.14 & 0.15 \\
$\Delta E_{S F}^{(1)}$ & -0.89 & -0.39 & -0.25 \\
\hline
\end{tabular}

${ }^{a}$ The $S_{2}$ structural optimizations and ZPE calculations are carried out with $C_{i}$ symmetry, in which the $S_{2}$ and $S_{1}$ states belong to different irreducible representations. Therefore, $S_{1}-S_{2}$ root flipping does not occur in the course of $S_{2}$ optimizations; ${ }^{b}$ for the (F) dimer, this is actually the vertical $E\left(S_{1}\right)$. We use $S_{2}$ to label this state for comparing its energy with the energies of the truly vertical $S_{2}$ states with the same HOMO-to-LUMO excitation scheme in the CAAC dimer and the (TFM) dimer; ${ }^{c}$ calculated at $S_{0}$ structure.

significant structural change between the $S_{0}$ and $T_{1}$ states.

The $S_{1}$ state arises from the single electron HOMO-to-LUMO+1 excitation. The planar framework remains in the $S_{1}$ optimized structure, with $d_{\mathrm{NCCN}}=175^{\circ}$. At the BHHLYP/Def2-SVP level, the HOMO-LUMO gap is calculated to be $3.51 \mathrm{eV}$, not significantly lower than the $3.91 \mathrm{eV}$ HOMO-LUMO+1 gap. Please note that the orbital energies are obtained in a restricted open-shell BHHLYP calculation for the lowest quintet state at the $S_{0}$ structure, which treats $\mathrm{HOMO}-1$ to $\mathrm{LUMO}+1$ equally. Their orbital energies are hence comparable. The HOMO and LUMO are compact and they are both out-of-plane $\pi$ orbitals. They hence have a substantial spatial overlap, which results in a large exchange integral that gives a high singlet excited state with one electron in each orbital. Please note that the large orbital overlap favors a low-lying triplet state. On the contrary, the HOMO and LUMO+1 (Figure 2(f)) are perpendicular and have a smaller exchange integral that can offset their $0.4 \mathrm{eV}$ orbital gap. Consequently, the HOMO-to-LUMO+1 singlet excitation 

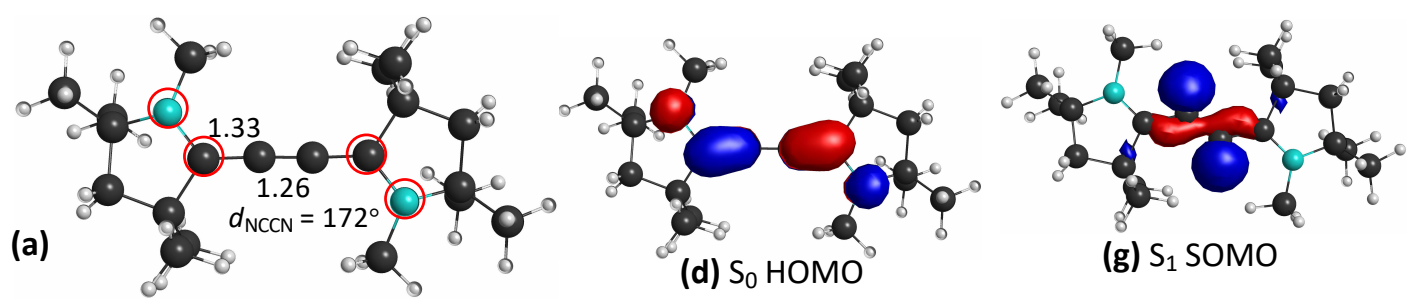

(d) $\mathrm{S}_{0} \mathrm{HOMO}$

(g) $\mathrm{S}_{1} \mathrm{SOMO}$
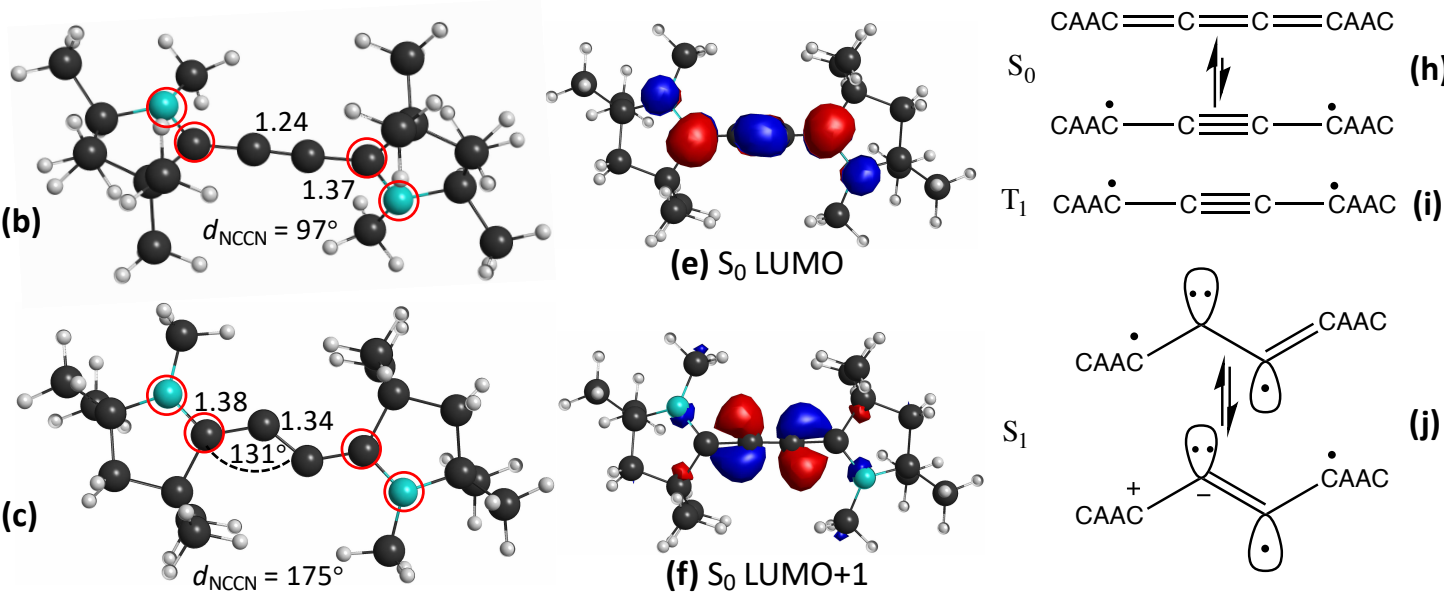

FIG. 2. The optimized structures of the (a) $S_{0}$, (b) $T_{1}$, and (c) $S_{1}$ states of $(\mathrm{CAAC})_{2}-\mathrm{C}_{2} ;(\mathrm{d})-(\mathrm{g})$ frontier orbitals of the structures of the denoted states; (h)-(i) typical resonance structures of the three states. Some important structural parameters are shown in panels (a)-(c), where the bond lengths are given in $\AA$ and the four atoms that define the dihedral angles are highlighted by red circles. In panel (i), only one set of resonance structures are shown for the $S_{1}$ state. There is another set which includes the symmetry images of the shown structures.

is of lower energy, while the HOMO-to-LUMO excitation gives the $S_{2}$ state. The vertical excitation energies of the two singlet states are close. With ZPE corrections, they are both $3.71 \mathrm{eV}$.

We cannot reproduce the non-ZPE-corrected $2.88 \mathrm{eV}$ vertical $E\left(S_{1}\right)$ of $(\mathrm{CAAC})_{2}-\mathrm{C}_{2}$ reported in Ref. 44. We took the structure from the SI of the paper and averaged the lowest five singlet states (employing the same state average scheme in the reference) in performing NEVPT2 calculation for the structure. The vertical $E\left(S_{1}\right)$ is calculated to be $3.13 \mathrm{eV}$, with $f=0.58$. This $f$ is close to the 0.6 reported in Ref. 44 . This $S_{1}$ state corresponds to the HOMO-to-LUMO excitation. The HOMO-to-LUMO+1 excitation gives the $S_{2}$ state, with the non-ZPE-corrected vertical $E\left(S_{1}\right)=3.60 \mathrm{eV}$. There are two factors that contribute to the differences between the results in Ref. 44 and ours. (1) Different active spaces are 
used. A smaller 4 electrons in 4 orbitals active space was used in Ref. 44, while the full valence $\pi$ active space with 8 orbitals and 10 electrons are employed in the present work. As mentioned in Section II, the calculated excitation energies of the $\mathrm{C}_{2}$-connected dimers (and the $\mathrm{C}_{4}$-connected dimers too) are highly sensitive to the active space, varying by tenths of eV. (2) Different dimer structures were used. The 1.33 and $1.26 \AA \mathrm{CC}$ bond lengths demonstrated in Figure 2(a) are in good agreement with the experimental values. The corresponding bond lengths of the structure used in Ref. 44 are 1.44 and $1.18 \AA$.

The $S_{1}$-optimized structure features an in-plane bending of the cumulene moiety, resulting in the $131^{\circ} \angle \mathrm{CCC}$ shown in Figure 2(c). The in-plane $\pi^{*}$ antibonding $\mathrm{LUMO}+1$ at the $S_{0}$ structure has evolved to the nonbonding singly occupied molecular orbital (SOMO) at the $S_{1}$ structure (Figure $2(\mathrm{~g})$ ). Correspondingly, the in-plane $\pi$ bonding orbital has also evolved to a similar nonbonding orbital. There is thus little in-plane $\pi$ bonding interaction between the two central $\mathrm{C}$ atoms. The $S_{1}$ state adopts the resonance structures shown in Figure $2(\mathrm{j})$, with three electrons distributed in the two in-plane $s p^{2}$ hybridized non-bonding orbitals. The ZPE-corrected vertical and adiabatic $E\left(S_{1}\right)$ s are calculated to be 3.71 and $2.15 \mathrm{eV}$. The significant structural change in the $S_{1}$ state is commensurate with the $1.56 \mathrm{eV}$ large reorganization energy. The perpendicular $\mathrm{HOMO}$ and $\mathrm{LUMO}+1$ give the 0.002 small oscillator strength $(f)$ for the vertical $S_{0}$-to- $S_{1}$ excitation. The $2.15 \mathrm{eV}$ adiabatic $E\left(S_{1}\right)$ and the $3.71 \mathrm{eV}$ vertical $E\left(S_{1}\right)$ are in fair agreement with the $\mathrm{UV} / \mathrm{Vis}$ spectrum of the dimer in $\mathrm{CH}_{2} \mathrm{Cl}_{2}$ solution. ${ }^{74}$ The spectrum consists of a structureless band, whose low energy edge is at $2.32 \mathrm{eV}(535 \mathrm{~nm})$ and with very low intensity. The intensity of the band increases sharply as the absorption energy increases to $3.65 \mathrm{eV}(340 \mathrm{~nm})$, beyond which the absorption was not recorded. The $340 \mathrm{~nm}$ absorption is so intense that it must involve the excitation to $S_{2}$, whose adiabatic and vertical excitation energies are 3.50 and $3.71 \mathrm{eV}$ (354 and $\left.334 \mathrm{~nm}\right)$, respectively, and $f=0.76$.

With the $C_{i}$ symmetry of the dimer in crystal, ${ }^{73,74}$ the HOMO-to-LUMO+1 excitation to the $S_{1}$ state is symmetry forbidden. In solution, the dimer can also adopt $C_{2}$ symmetry, as the $C_{2}$ configuration is only higher by $0.002 \mathrm{eV}$ (estimated at the NEVPT2 level) than the $C_{i}$ configuration. Although the transition is now not symmetry-forbidden, as mentioned above, the small $f=0.002$ arises from the transition between two perpendicular $\pi$ orbitals. Despite the small (strictly zero) oscillator strength in $C_{2}\left(C_{i}\right)$ symmetry, the transition may be turned on by vibrations, and one possible vibrational mode is the torsional motion of 
the CAAC units about the $\mathrm{C}_{2}$ spacer. Such an out-of-plane vibration can induce mixing of the out-of-plane LUMO and the in-plane LUMO+1, and hence turns on the HOMO-toLUMO+1 transition. According to our MRSF-TDDFT calculation, the frequency of this mode is only $40 \mathrm{~cm}^{-1}$ low. Out-of-plane wagging and rocking modes of the CAAC units with respect to the spacer are of similarly low frequencies. These vibrations can be thermally activated. Overall, the structure of the dimer is not rigid and vibration-induced $S_{0}$-to- $S_{1}$ transition is possible. Other than the symmetry-breaking vibrations, the $S_{0}$-to- $S_{1}$ transition intensity may also be enhanced by the surrounding $\mathrm{CH}_{2} \mathrm{Cl}_{2}$ polar solvent molecules. The mixing of the perpendicular LUMO and $\mathrm{LUMO}+1$ can be induced by an electric dipole pointing in a direction between them. The conjecture of the vibration- and solvent-induced $S_{0}$-to- $S_{1}$ transition is consistent with the low intensity of the absorption at $2.32 \mathrm{eV}$ (535 $\mathrm{nm})$.

The excited states of $(\mathrm{CAAC}(\mathrm{TFM}))_{2}-\mathrm{C}_{2}$ and $(\mathrm{CAAC}(\mathrm{F}))_{2}-\mathrm{C}_{2}$ feature similar excitation schemes and take similar resonance and geometrical structures for those states. Their higher diradical characters are reflected by their lower vertical $E\left(T_{1}\right)$ s of 2.33 and $2.19 \mathrm{eV}$ and their larger $n_{\mathrm{LUNO}}$ s of 0.14 and 0.15 . The order of the diradical character follows the expectation based on the stabilization of the CAAC $\pi$ LUMO by the trifluoromethylation and fluorination at the $\alpha \mathrm{C}$. With structural reorganization, the adiabatic $E\left(T_{1}\right)$ s are calculated to be 1.43 and $1.29 \mathrm{eV}$.

The $S_{1}$ state has little to do with the diradical character, and the ZPE-corrected vertical $E\left(S_{1}\right) \mathrm{s}$ of the $(\mathrm{TFM})$ and $(\mathrm{F})$ dimers are calculated to be 3.66 and $3.71 \mathrm{eV}$, which are close to the $3.71 \mathrm{eV}$ of the unsubstituted structure. After the $S_{1}$ structures are optimized, the adiabatic $E\left(S_{1}\right) \mathrm{s}$ are 2.47 and $2.33 \mathrm{eV}$, larger than the $2.15 \mathrm{eV}$ of the unsubstituted structure. The higher $E\left(S_{1}\right)$ s leave more room for the substituted dimers to satisfy Eq. 1. Please note that at the $S_{0}$ structure of the $(\mathrm{F})$ dimer, the HOMO-to-LUMO+1 excitation requires slightly higher energy than the HOMO-to-LUMO excitation (3.71 vs. $3.53 \mathrm{eV}$, also see the $b$ note in Table I). The HOMO-to-LUMO+1 excited state becomes lower in energy in the course of the $S_{1}$ structural optimization and gives the adiabatic $S_{1}$ state. For this reason and also the convenience to compare with the results of (CAAC)- $\mathrm{C}_{2}$ and the (TFM) dimer, we still label the HOMO-to-LUMO+1 excitation at the $S_{0}$ structure the $S_{1}$ state, while the HOMO-to-LUMO excitaiton the $S_{2}$ state.

With the ZPE-corrected adiabatic energies, $\Delta E_{S F}^{(1)}=E\left(S_{1}\right)-2 E\left(T_{1}\right)=-0.89,-0.39$, 
and $-0.25 \mathrm{eV}$ for $(\mathrm{CAAC})_{2}-\mathrm{C}_{2}$, the $(\mathrm{TFM})$, and the $(\mathrm{F})$ dimers. None of them meets the primary criterion Eq. 1 for SF chromophores, although the trend is in the right direction with the TFM and F substitutions. In addition to the energy mismatches, these molecules have another disadvantage as SF chromophores. Their $>3.6 \mathrm{eV}$ vertical $E\left(S_{1}\right) \mathrm{s}$ are in the UV region, in which the solar spectrum has low photon flux density. And the $S_{1}$ states have negligible oscillator strengths. The $S_{2}$ states involve the HOMO-to-LUMO excitation and their oscillator strengths are more substantial. Their vertical excitation energies are deeper in the UV region (except for the $(\mathrm{F})$ dimer, whose vertical $E\left(S_{2}\right)$ is at the border of the UV and Vis regions). Overall, the three CAAC dimers do not have good absorption of the solar spectrum. The importance of structural reorganization of the excited states is clearly shown in this series of calculations. If we compare the vertical $E\left(S_{1}\right) \mathrm{s}$ and the adiabatic $E\left(T_{1}\right) \mathrm{s}$, all three molecules satisfy Eq. 1 . The $>1.19 \mathrm{eV}$ large reorganization energies of the $S_{1}$ states, however, reverse the signs of the respective $\Delta E_{S F}^{(1)} \mathrm{s}$.

\section{CAAC Dimers with a $\mathrm{C}_{4}$ Spacer}

The HOMO and LUMO in Figure 2(d) and (e) show that the $\pi$ LUMOs of the CAAC units have (anti)bonding interactions with the LUMO (HOMO) of the $C_{2}$ spacer. Without these interactions, each of the CAAC $\pi$ LUMO would hold an unpaired electron in the dimer, and the dimer would be a pure diradical. The bonding and antibonding interactions open up the HOMO-LUMO gap and reduce the diradical character, so that the dimer does not satisfy Eq. 1. A longer spacer, with its attenuated HOMO and LUMO amplitudes at the connecting sites with the CAAC units, is likely to reduce the bonding/antibonding interactions and increase the diradical character, making the dimer satisfy Eq. 1. Following this logic, we increase the length of the spacer from $\mathrm{C}_{2}$ to $\mathrm{C}_{4}$. The calculated results of the $\mathrm{C}_{4}$-connected dimers are summarized in Table II.

The $S_{0}, T_{1}$, and $S_{1}$ structures of $(\mathrm{CAAC})_{2}-\mathrm{C}_{4}$ are shown in Figure $3(\mathrm{a})$-(c), which share geometrical features with the $\mathrm{C}_{2}$ analogues. This is reasonable given the similarity in electronic structure of cumulenes with different lengths. While the HOMO in Figure 3(d) shows bonding interaction between the CAAC fragments and the spacer, the LUMO+1 in Figure 3(f), which corresponds to the LUMO in Figure 2(e), shows largely nonbonding character. The LUMO and LUMO+1 are almost degenerate, with the 2.53 and $2.62 \mathrm{eV}$ HOMO-LUMO and 

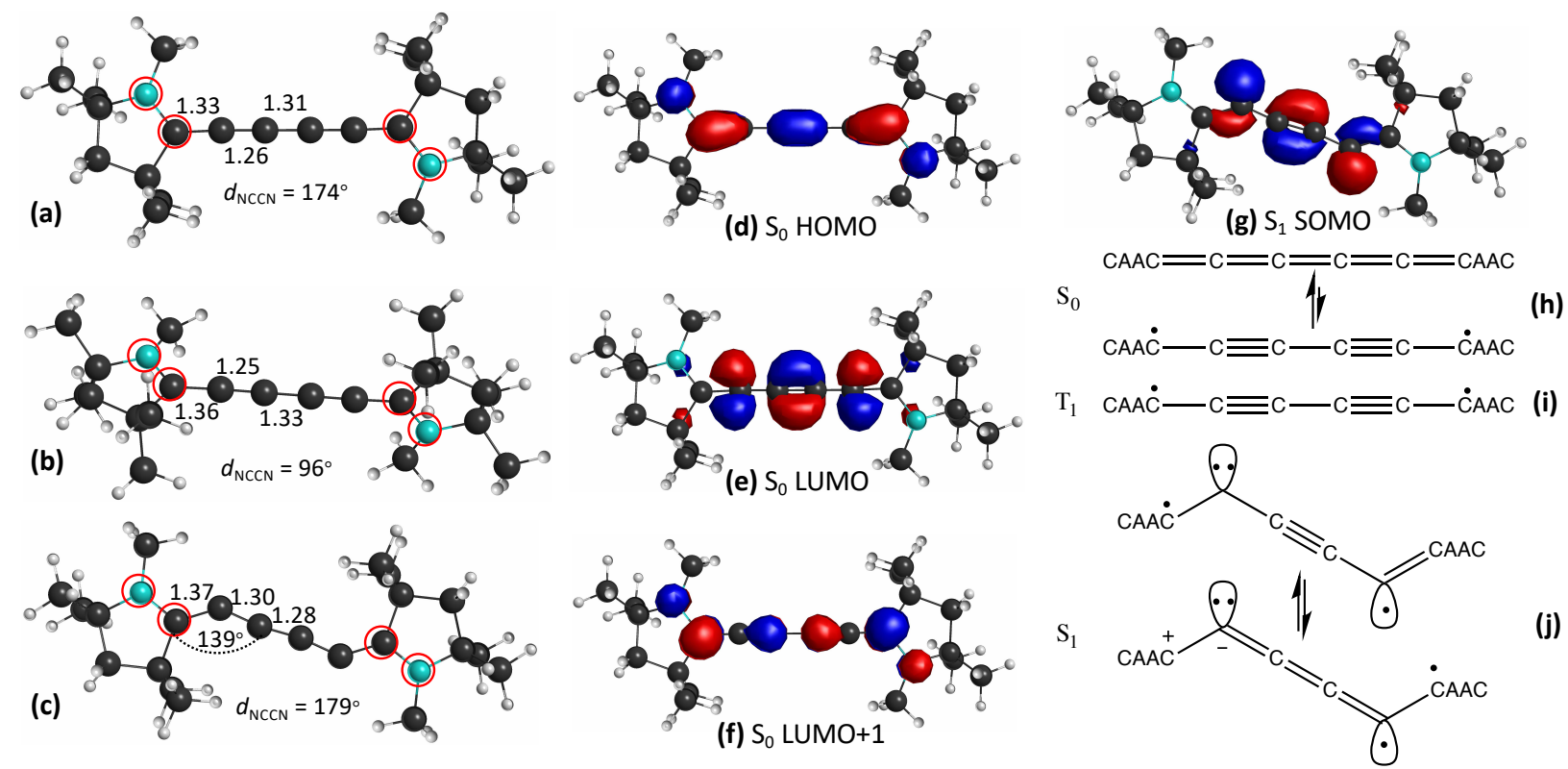

FIG. 3. The optimized structures of the (a) $S_{0}$, (b) $T_{1}$, and (c) $S_{1}$ states of $(\mathrm{CAAC})_{2}-\mathrm{C}_{4} ;(\mathrm{d})-(\mathrm{g})$ frontier orbitals of the structures of the denoted states; (h)-(j) typical resonance structures of the three states. Some important structural parameters are shown in panels (a)-(c), where the bond lengths are given in $\AA$ and the four atoms that define the dihedral angles are highlighted by red circles. In panel $(\mathrm{j})$, only one set of resonance structures are shown for the $S_{1}$ state. There is another set which includes the symmetry images of the shown structures.

HOMO-LUMO+1 gaps at the BHHLYP level of calculation. The $T_{1}$ and $S_{1}$ states involve the same excitations as in the $\mathrm{C}_{2}$-connected dimers, except that the LUMO and LUMO+1 of the $\mathrm{C}_{4}$-connected dimer correspond to the LUMO+1 and LUMO of the $\mathrm{C}_{2}$ analogue. The vertical $E\left(T_{1}\right) \mathrm{s}$ are $1.80,1.65$, and $1.58 \mathrm{eV}$ for $(\mathrm{CAAC})_{2}-\mathrm{C}_{4},(\mathrm{CAAC}(\mathrm{TFM}))_{2}-\mathrm{C}_{4}$, and $(\mathrm{CAAC}(\mathrm{F}))_{2}-\mathrm{C}_{4}$, respectively, and the ground state $n_{\mathrm{LUNO}}$ are $0.19,0.20$, and 0.21. Both sets of data indicate the stronger diradical characters brought by the $\mathrm{C}_{4}$ spacer, and brought by the trifluoromethylation and fluorination. The pure diradical character of the $T_{1}$ state results in the structural reorganization to the perpendicular arrangement of the CAAC fragments as shown in Figure 3(b), along with a $0.74 \mathrm{eV}$ substantial reorganization energy.

In the $S_{1}$ state the antibonding LUMO is occupied and evolves to the SOMO at the $S_{1}$ structure (Figure $3(\mathrm{~g})$ ) following the bending of the $\mathrm{C}_{6}$ cumulene fragment. The SOMO exhibits nonbonding character at the two ends of the spacer. The $0.79 \mathrm{eV} S_{1}$ reorganization energy is similar to the $T_{1}$ analogue. The structures, orbitals, and excitation schemes of 
TABLE II. Calculated results for the three $\mathrm{C}_{4}$-connected dimers. All energies are given in $\mathrm{eV}$.

\begin{tabular}{cccc}
\hline & CAAC & $(\mathrm{TFM})$ & $(\mathrm{F})$ \\
\hline Adiabatic $E\left(S_{1}\right)$ & 1.65 & 1.89 & 1.87 \\
Vertical $E\left(S_{1}\right)(f)$ & $2.44(0.0001)$ & $2.50(0.001)$ & $2.56(0.001)$ \\
Vertical $E\left(S_{2}\right)^{a}(f)$ & $3.33(0.71)$ & $3.19(0.61)$ & $3.16(0.62)$ \\
Adiabatic $E\left(T_{1}\right)$ & 1.06 & 0.98 & 0.91 \\
Vertical $E\left(T_{1}\right)$ & 1.80 & 1.65 & 1.58 \\
$E\left(T_{1}\right) @ S_{1} \mathrm{~g}$ & 1.40 & 1.82 & 1.65 \\
$n_{\text {LUNO }}^{b}$ & 0.19 & 0.20 & 0.21 \\
$\lambda^{c}$ & 1.08 & 1.51 & 1.41 \\
Barrier $^{d}$ & 0.56 & 0.41 & 0.33 \\
$\Delta E_{S F}^{(1)}$ & -0.47 & -0.07 & 0.05 \\
\hline
\end{tabular}

${ }^{a}$ Without ZPE correction; ${ }^{b}$ calculated at $S_{0}$ structure; ${ }^{c}$ reorganization energies for $S_{0} S_{1} \rightarrow T_{1} T_{1} ;{ }^{d}$ barrier for $S_{0} S_{1} \rightarrow T_{1} T_{1}$.

the $\mathrm{C}_{4}$-connected dimers are similar to those of the $\mathrm{C}_{2}$-connected dimers. We thus assign the similar resonance structures as shown in Figure $3(\mathrm{~h})-(\mathrm{j})$ to the three states of $(\mathrm{CAAC})_{2^{-}}$ $\mathrm{C}_{4}$. The structures and orbitals of the (TFM) and (F) dimers are similar to those of the unsubstituted dimer and are hence not shown in Figure 3.

The $S_{1}$ and $T_{1}$ energies of the three $\mathrm{C}_{4}$-connected dimers, both vertical and adiabatic, are lower than the $\mathrm{C}_{2}$ counterparts. This is consistent with the smaller HOMO-to-LUMO and HOMO-to-LUMO+1 gaps brought by the longer length of the molecules. With the adiabatic $E\left(S_{1}\right) \mathrm{s}$ and $E\left(T_{1}\right) \mathrm{s}, \Delta E_{S F}^{(1)}=-0.47,-0.07$, and $0.05 \mathrm{eV}$. The (TFM) dimer is close to satisfy and the $(\mathrm{F})$ dimer satisfies Eq. 1. The $0.07 \mathrm{eV} \mathrm{SF}$ endoergicity of the (TFM) dimer can be overcome by thermal activation. Even for the unsubstituted structure, the subtraction results in a less negative value than the $\mathrm{C}_{2}$ analogue. With the smaller orbital gaps, the vertical excitation energies of the lowest light-absorbing singlet excited state $E\left(S_{2}\right)$ s are $3.33,3.19$, and $3.16 \mathrm{eV}$ for the three $\mathrm{C}_{4}$-connected dimers, all smaller than the corresponding energies of the $\mathrm{C}_{2}$-connected dimers. The $S_{2}$ state is dominated by the HOMO-to-LUMO+1 single electron excitation and has large oscillator strength: $f=0.71$, 
0.61, and 0.62 for the three dimers. Those absorptions occur in the visible region of the solar spectrum, which has higher photon flux density than the UV region for the $\mathrm{C}_{2}$-connected dimers. The $E\left(S_{2}\right)$ s above do not contain ZPE corrections. They are higher than the corresponding $E\left(S_{1}\right)$ s by $0.5 \mathrm{eV}$, and the ZPE corrections would not alter the $S_{1}-S_{2}$ energy ordering. We hence do not optimize the $S_{2}$ structures and calculate their ZPEs. On the contrary, the vertical $E\left(S_{1}\right)$ and $E\left(S_{2}\right)$ s of the $\mathrm{C}_{2}$-connected dimers are close and it is necessary to include ZPE corrections to clarify the energy ordering.

Overall, the improvement by replacing the $\mathrm{C}_{2}$ spacer by the $\mathrm{C}_{4}$ is evident. However, it is too early to conclude that $(\mathrm{CAAC}(\mathrm{TFM}))_{2}-\mathrm{C}_{4}$ and $(\mathrm{CAAC}(\mathrm{F}))_{2}-\mathrm{C}_{4}$ are good candidates for SF chromophores. The substantial structural reorganizations in the $S_{0}$-to- $S_{1}$ and $S_{0}$-to$T_{1}$ excitations and the significant structural differences between the $S_{1}$ - and $T_{1}$-optimized structures imply large reorganization energies $(\lambda \mathrm{s})$ for the $S_{0} S_{1} \rightarrow T_{1} T_{1}$ processes in the two dimers. $\lambda$ can be approximated as

$$
\begin{aligned}
\lambda & =E\left(T_{1}\right) @ S_{0} \mathrm{~g}+E\left(T_{1}\right) @ S_{1} \mathrm{~g}-2 E\left(T_{1}\right) @ T_{1} \mathrm{~g} \\
& =E\left(T_{1}\right) @ S_{0} \mathrm{~g}+E\left(T_{1}\right) @ S_{1} \mathrm{~g}-E\left(S_{1}\right) @ S_{1} \mathrm{~g}+\Delta E_{S F}^{(1)} .
\end{aligned}
$$

Please note that $E\left(S_{0}\right) @ S_{0}$ g has been defined to be the 0 reference. For the $\mathrm{C}_{4}$-connected dimers, the $T_{1}$ states at the $S_{1}$ structures have the same occupation scheme as the corresponding $S_{1}$ states. The different $T_{1}$ occupation schemes compared to those in the $S_{0}$ structures are a reasonable result of the stabilization of the $S_{1}$ SOMO shown in Figure $3(\mathrm{~g})$. With the excitation energies reported in Table II, $\lambda$ is calculated to be 1.08, 1.51 and $1.41 \mathrm{eV}$ for the three $\mathrm{C}_{4}$-connected dimers. According to the Marcus Theory ${ }^{76}$, the barrier for the fission can be estimated as $\frac{\left(\lambda-\Delta E_{S F}^{(1)}\right)^{2}}{4 \lambda}$. For the three dimers, the barriers are estimated to be 0.56, 0.41 and $0.33 \mathrm{eV}$, respectively. They are fairly high for a photo-induced process such as $\mathrm{SF}$, recalling the limited life times of the $S_{1}$ states. Although the (TFM) and (F) dimers satisfy the thermodynamics criterion Eq. 1, SFs are not likely to occur in those systems due to kinetics unfavorability. Please note that we only use Marcus Theory to estimate the SF barriers in this paper, without any intention to claim that the fissions, if they occur, follow the perturbation mechanism of the theory.

The large barriers arise from the significant structural reorganizations in the $T_{1}$ states from the $S_{0}$ and $S_{1}$ structures to the $T_{1}$ structure. The $\mathrm{C}_{2}$ and $\mathrm{C}_{4}$ spacers are cylindrically symmetric, and so are their valence $\pi$ systems. The cylindrical $\pi$ systems allow the two 
(a)

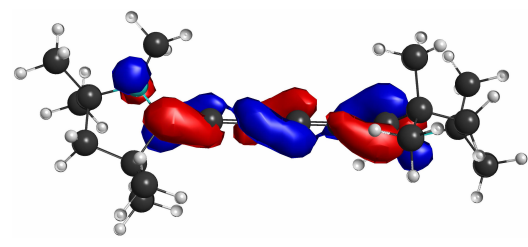

(b)

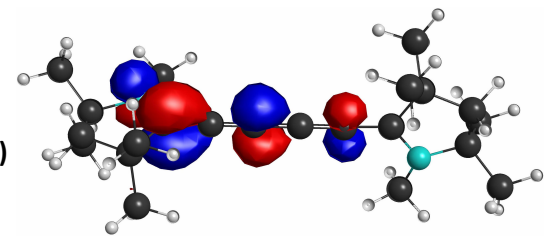

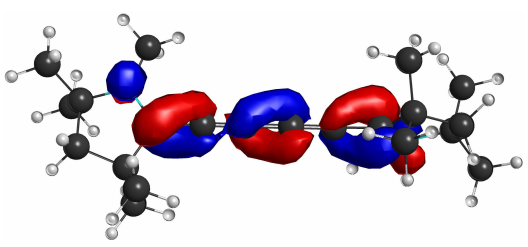

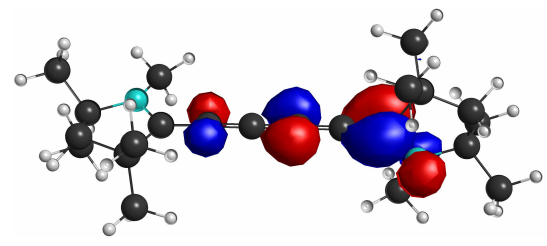

FIG. 4. (a) The two singly occupied molecular orbitals $\mathrm{SOMO}_{1}$ and $\mathrm{SOMO}_{2}$ in the $T_{1}$ state of $(\mathrm{CAAC})_{2}-\mathrm{C}_{4}$ and $(\mathrm{b})$ the corresponding localized orbitals obtained from $\frac{1}{\sqrt{2}}\left(\mathrm{SOMO}_{1} \pm \mathrm{SOMO}_{2}\right)$, which are perpendicular $\pi$ orbitals.

CAAC units to take a perpendicular configuration along the spacers, so that the SOMOs of the $T_{1}$ states are perpendicular to each other and change from bonding and antibonding orbitals in the $S_{0}$ structure to nonbonding orbitals in the $T_{1}$ structure (Figure 4 ). Our calculation shows that each of the SOMOs in Figure 4(a) contributes $\leq 0.06$ Mayer bond order between adjacent $\mathrm{C}$ atoms along the central linear fragment of the molecule. The nonbonding nature of the SOMOs is evident. These nonbonding SOMOs lower $E\left(T_{1}\right)$ along the coplanar-to-perpendicular structural reorganization, just as in the case of the triplet ethylene. Please note that by "coplanar" and "perpendicular", we only consider the configuration of the $\mathrm{N}$, the carbene $\mathrm{C}$, and the $\alpha \mathrm{C}$ in the two CAAC units, and the spacer. The significant $T_{1}$ structural reorganization has two opposite effects for $(\mathrm{CAAC}(\mathrm{TFM}))_{2}-\mathrm{C}_{4}$ and $(\mathrm{CAAC}(\mathrm{F}))_{2}-\mathrm{C}_{4}:(1)$ it lowers their adiabatic $E\left(T_{1}\right)$ s so that Eq. 1 is satisfied or closed to be satisfied; (2) it raises the reorganization energies and also the barriers in SF, so that the two dimers are unlikely to undergo SF. The cylindrical $\pi$ systems consist of perpendicular (out-of-plane and in-plane) $\pi$ orbitals in the $S_{0}$ structures. The $S_{1}$ states arise from electron transition from out-of-plane orbital to in-plane orbital. The antibonding interaction of the singly occupied in-plane $\pi^{*}$ orbital is mitigated by the in-plane bending of the $S_{1}$ structure, which enhances the nonbonding character of the orbital. In short, the cylindrical $\pi$ systems of the $\mathrm{C}_{2}$ and $\mathrm{C}_{4}$ spacers are responsible for their large $S_{1}$ and $T_{1}$ reorganization energies.

Knowing the kinetics unfavorability, we do not need to consider Eq. 2 for the $\mathrm{C}_{4}$-connected dimers. We mention in passing that the optimizations of $T_{2}$ structures of the $\mathrm{C}_{2^{-}}$and $\mathrm{C}_{4^{-}}$ 
connected dimers failed to converge. This is because the $T_{2}$ states have the same ${ }^{3} B$ symmetry as the $T_{1}$ states and the two states exchange their characters (i.e., root flipping) during the optimization. The $T_{2}$ states of the $\mathrm{C}_{2}$-connected dimers correspond to the HOMO-toLUMO+1 excitation, and $\mathrm{C}_{4}$ analogues correspond to the HOMO-1-to-LUMO excitation. The HOMO-1 of $(\mathrm{CAAC})_{2}-\mathrm{C}_{4}$ is shown in Figure $\mathrm{S} 1$ in $\mathrm{SI}$.

\section{CAAC Dimers with a Para-Phenylene Spacer}

To avoid the problem induced by the cylindrical $\pi$ system of the spacer, we select the p-phenylene (abbreviated as $\mathrm{Ph}$ ) as the spacer to connect two CAAC moieties. Unlike the sequence of presentation in the previous sections, we first focus on the results of $(\mathrm{CAAC}(\mathrm{F}))_{2^{-}}$ $\mathrm{Ph}$ and $(\mathrm{CAAC}(\mathrm{TFM}))_{2}-\mathrm{Ph}$. This is because the optimization of the $S_{1}$ state of $(\mathrm{CAAC})_{2}-\mathrm{Ph}$ failed (vide infra).

The $S_{0^{-}}, T_{1^{-}}$, and $S_{1^{-}}$pptimized structures of $(\mathrm{CAAC}(\mathrm{F}))_{2}-\mathrm{Ph}$ are shown in Figure $5(\mathrm{a})$, (b), and (c). The calculated results are summarized in Table III. They look more similar to each other than the $\mathrm{C}_{2}$ and $\mathrm{C}_{4}$ analogues. Especially, the key dihedral angle changes by up to $20^{\circ}$ only. Here, we choose the dihedral angle made by the CAAC unit and the phenyl spacer $\left(d_{\mathrm{NCCC}}\right)$ to indicate the coplanarity of the central fragment of the dimer. The smaller changes in the structure are consistent with the smaller $S_{1}$ and $T_{1}$ reorganization energies, which are 0.20 and $0.54 \mathrm{eV}$, respectively, vs. the 0.69 and $0.67 \mathrm{eV}$ of $(\mathrm{CAAC}(\mathrm{F}))_{2^{-}}$ $\mathrm{C}_{4}$. The (TFM) dimer shares similar structures with $(\mathrm{CAAC}(\mathrm{F}))_{2}-\mathrm{Ph}$ and thus also has smaller $S_{1}$ and $T_{1}$ reorganization energies than its $\mathrm{C}_{4}$ counterpart. The replacement of $\mathrm{C}_{4}$ by $p$-phenylene does point in the right direction.

The $1.53 \mathrm{eV}$ vertical $E\left(T_{1}\right)$ of $(\mathrm{CAAC}(\mathrm{F}))_{2} \mathrm{Ph}$ is similar to the $1.58 \mathrm{eV}$ of $(\mathrm{CAAC}(\mathrm{F}))_{2^{-}}$ $\mathrm{C}_{4}$. This suggests similar diradical characters of the two dimers. The $0.18 n_{\mathrm{LuNO}}$ of $(\mathrm{CAAC}(\mathrm{F}))_{2}-\mathrm{Ph}$, however, suggests a lower diradical character. This inconsistence is attributed to the difference in the active spaces in use. For $(\mathrm{CAAC}(\mathrm{F}))_{2}-\mathrm{C}_{4}$, the in-plane $\pi$ electrons can be excited and contribute to the higher $0.21 n_{\mathrm{LUNO}}$. Similar in-plane $\pi$ electrons are not present in $(\mathrm{CAAC})_{2}-\mathrm{Ph}$. It is more reasonable to compare $n_{\mathrm{LUNO}}$ s from calculations with comparable active spaces, e.g., those of the $\mathrm{C}_{2^{-}}$and $\mathrm{C}_{4}$-connected dimers. We hence take the vertical $E\left(T_{1}\right)$ as a more reliable indicator to compare the diradical characters of $(\mathrm{CAAC}(\mathrm{F}))_{2}-\mathrm{Ph}$ and $(\mathrm{CAAC}(\mathrm{F}))_{2}-\mathrm{C}_{4}$. 

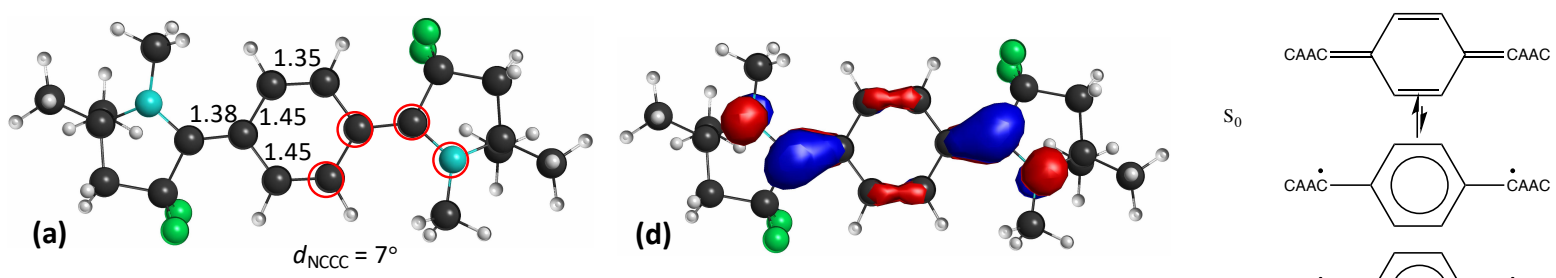

(g)
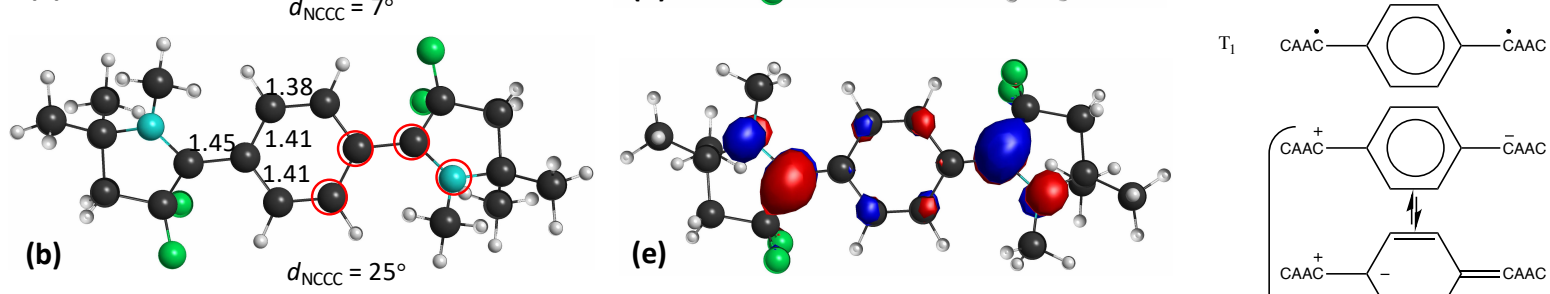

(h)
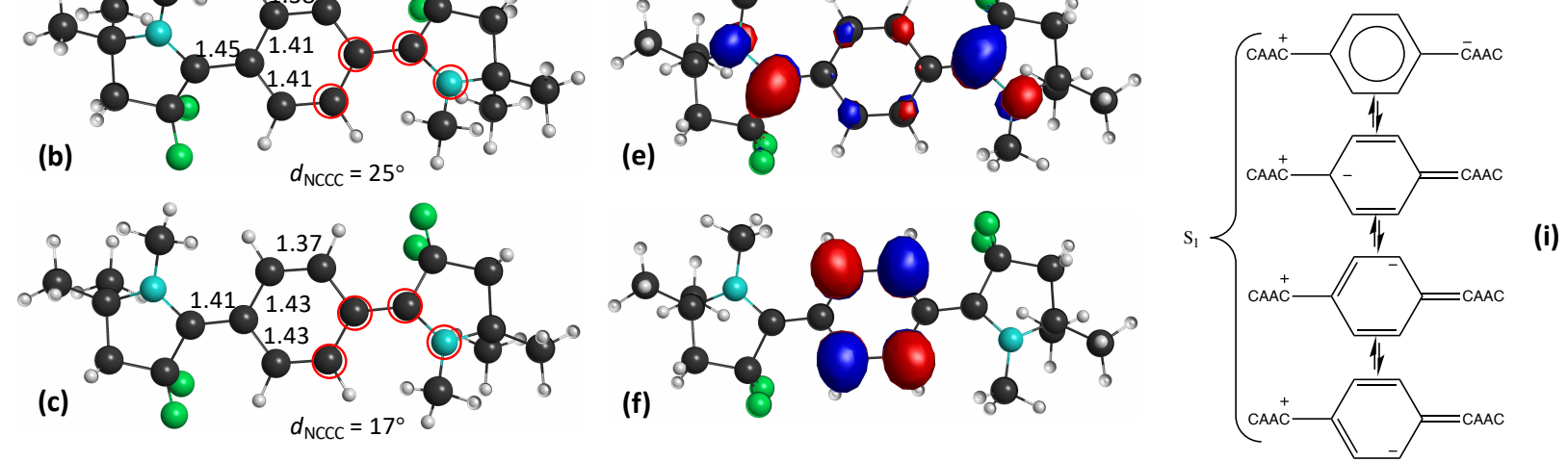

FIG. 5. The optimized structures of the (a) $S_{0}$, (b) $T_{1}$, and (c) $S_{1}$ states of $(\mathrm{CAAC}(\mathrm{F}))_{2}$-Ph; (d)-(f) frontier orbitals of the structures of the $S_{0}$ state; (g)-(i) typical resonance structures of the three states. Some important structural parameters are shown in panels (a)-(c), where the bond lengths are given in $\AA$ and the four atoms that define the dihedral angles are highlighted by red circles. In panel (i), only some resonance structures are shown for the $S_{1}$ state. The others include those with the + and - charges being swapped and those with the symmetry images.

$(\mathrm{CAAC}(\mathrm{F}))_{2}-\mathrm{Ph}$ satisfies Eq. 1, with $\Delta E_{S F}^{(1)}=0.62 \mathrm{eV}$. Both the $S_{1}$ and $T_{1}$ states correspond to the HOMO-to-LUMO excitation, and the HOMO and LUMO are shown in Figure 5(d) and (e). The HOMO (LUMO) arises from (anti)bonding interaction between the LUMO (HOMO) of the phenylene spacer and the two $\pi$ orbitals of the carbenes. $(\mathrm{CAAC}(\mathrm{F}))_{2}-\mathrm{Ph}$ bears a typical $p$-quinodimethane core structure, and its diradical character is associated to the $S_{0}$ resonance structure with an aromatic phenylene (see the $S_{0}$ resonance structures in Figure $5(\mathrm{~g}))$. The $S_{0}$ state is dominated by the resonance structure with alternating CC single and double bonds, as reflected by the bond lengths shown in Figure 5(a). The $T_{1}$ state simply takes the diradical resonance structure associated with the phenylene spacer in its aromatic form. Correspondingly, the CC bond lengths of the phenylene are more equalized in this state, and the $\mathrm{CC}$ bonds that connect the CAAC units and the spacer are elongated from 1.38 to $1.45 \AA$ (i.e., from double bonds between $s p^{2} \mathrm{C}$ atoms to single 
TABLE III. Calculated results for the two substituted Ph-connected dimers. All energies are given in $\mathrm{eV}$.

\begin{tabular}{cccc}
\hline & CAAC & $(\mathrm{TFM})$ & $(\mathrm{F})$ \\
\hline Adiabatic $E\left(S_{1}\right)$ & $2.45^{a}$ & 2.31 & 2.60 \\
Vertical $E\left(S_{1}\right)(f)$ & $2.59(0.83)$ & $2.45(0.70)$ & $2.70(0.85)$ \\
Adiabatic $E\left(T_{1}\right)$ & 1.24 & 0.94 & 0.99 \\
Vertical $E\left(T_{1}\right)$ & 1.63 & 1.41 & 1.53 \\
$E\left(T_{1}\right) @ S_{1} \mathrm{~g}$ & $1.34^{a}$ & 1.13 & 1.20 \\
Adiabatic $E\left(T_{2}\right)$ & 2.68 & 3.18 & 3.07 \\
$n_{\text {LUNO }}^{b}$ & 0.16 & 0.20 & 0.18 \\
$\lambda^{c}$ & 0.49 & 0.66 & 0.75 \\
Barrier $^{d}$ & 0.14 & 0.02 & 0.02 \\
$\Delta E_{S F}^{(1)}$ & -0.03 & 0.43 & 0.62 \\
$\Delta E_{S F}^{(2)}$ & 0.20 & 1.30 & 1.09 \\
\hline
\end{tabular}

${ }^{a}$ The adiabatic $E\left(S_{1}\right)$ and $E\left(T_{1}\right) @ S_{1}$ g of the CAAC dimer is for the $S_{1}$-optimized structure with $C_{2}$ symmetry, which has a an imaginary frequency. See the discussion in the text; ${ }^{b}$ calculated at $S_{0}$ structure; ${ }^{c}$ reorganization energies for $S_{0} S_{1} \rightarrow T_{1} T_{1} ;{ }^{d}$ barrier for $S_{0} S_{1} \rightarrow T_{1} T_{1}$.

bonds).

It is well known that the HOMO-to-LUMO one-electron singlet transition results in a charge resonance state with one of the localized orbitals in Figure 6 being doubly occupied while the other being empty. ${ }^{77}$ The charge resonance structures shown in Figure 5(i) correspond to that an electron is depleted from the CAAC on the left side, which contributes the most to the left localized orbital in Figure 6, and the electron is deposited in the $\mathrm{C}$ sites on the spacer and the other CAAC that contribute to the right localized orbital in the figure. The charge-resonance structures with the backward electron transfers and symmetry images of all these structures also contribute to the $S_{1}$ state, but are not shown in Figure 5(i).

The $T_{2}$ state of $(\mathrm{CAAC}(\mathrm{F}))_{2}$-Ph corresponds to the HOMO-to-LUMO+1 excitation (see Figure 5(f) for LUMO+1). The ZPE-corrected adiabatic $E\left(T_{2}\right)$ is calculated to be $3.07 \mathrm{eV}$, giving $\Delta E_{S F}^{(2)}=E\left(T_{2}\right)-2 E\left(T_{1}\right)=1.09 \mathrm{eV}$, satisfying Eq. 2. With $E\left(T_{1}\right) @ S_{1} \mathrm{~g}=1.20 \mathrm{eV}$, 


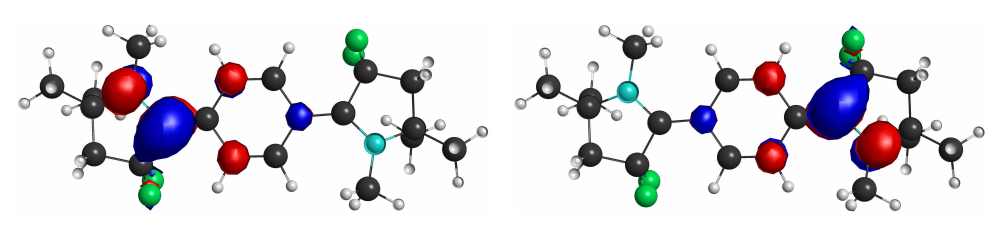

FIG. 6. Localized orbitals of $(\mathrm{CAAC}(\mathrm{F}))_{2}$ - $\mathrm{Ph}$ obtained from $\frac{1}{\sqrt{2}}(\mathrm{HOMO} \pm \mathrm{LUMO})$.

the reorganization energy $\lambda$ for the $S_{0} S_{1} \rightarrow T_{1} T_{1}$ fission is estimated to be $0.75 \mathrm{eV}$, and the barrier is estimated to be $0.02 \mathrm{eV}$. The less substantial structural reorganizations of the $S_{1}$ and $T_{1}$ states lead to the half smaller $\lambda$ compared to $(\mathrm{CAAC}(\mathrm{F}))_{2}-\mathrm{C}_{4}$. The smaller $\lambda$ together with the large positive $\Delta E_{S F}^{(1)}$ result in the essentially barrier-less SF kinetics. The vertical $E\left(S_{1}\right)$ is $2.70 \mathrm{eV}$ with $f=0.85$. It corresponds to an intense absorption at the visible region of the solar spectrum that has fairly high photon flux density. Another advantage of $(\mathrm{CAAC}(\mathrm{F}))_{2} \mathrm{Ph}$ is its $0.99 \mathrm{eV}$ adiabatic $E\left(T_{1}\right)$, which is close to the $\sim 1 \mathrm{eV}$ optimal band gap that maximizes efficiency of a SF-based photovoltaics device. ${ }^{1,4}$ With all these advantages, $(\mathrm{CAAC}(\mathrm{F}))_{2}-\mathrm{Ph}$ is a good candidate for $\mathrm{SF}$ chromophore.
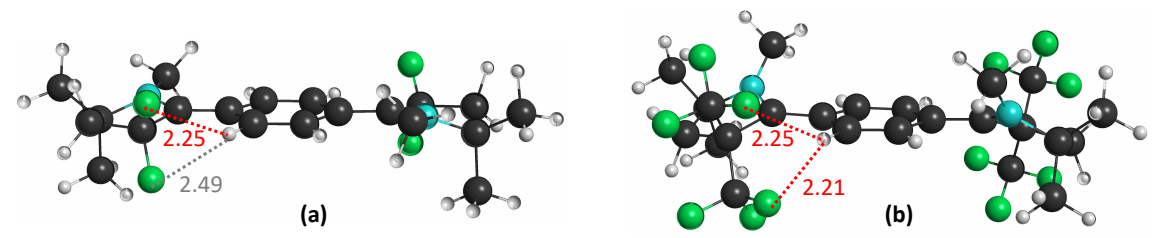

FIG. 7. Hydrogen bonds of $\mathrm{F}$ atoms in (a) $(\mathrm{CAAC}(\mathrm{F}))_{2}-\mathrm{Ph}$ and (b) $(\mathrm{CAAC}(\mathrm{TFM}))_{2}-\mathrm{Ph}$. The highlighted internuclear distances are given in $\AA$. The red dashed lines indicate $\mathrm{F} \cdots \mathrm{H}$ hydrogen bonds.

All those advantages are shared by the (TFM) dimer, which is thus another good candidate. It is interesting that the (TFM) dimer appears to have a higher diradical character than the $(\mathrm{F})$ dimer, indicated by its higher $n_{\mathrm{LUNO}}=0.20$ and lower vertical $E\left(T_{1}\right)=1.41 \mathrm{eV}$. This is inconsistent with the expectation based on the electronegativities of TFM and F. We attribute this anomaly to the $\mathrm{F} \cdots \mathrm{H}$ hydrogen bonds between the $\mathrm{F}$ atoms on the CAAC units and the $\mathrm{H}$ atoms on the spacer. One of the two highlighted F-H distances shown in Figure $7(\mathrm{a})$ for the $(\mathrm{F})$ dimer is shorter than the $2.26 \AA$ typical $\mathrm{F} \cdot \mathrm{H}$ bond distance between a $\mathrm{C}\left(s p^{3}\right)-\mathrm{F}$ and and a $\mathrm{C}\left(s p^{2}\right)-\mathrm{H}^{78}$ There is certainly $\mathrm{F} \cdots \mathrm{H}$ bonding interaction between the CAAC units and the spacer. As shown in Figure 7(b), there are two F-H 
distances between one CAAC unit and the spacer that are shorter than $2.26 \AA$. There are thus twice more F $\cdots \mathrm{H}$ hydrogen bonds in the (TFM) dimer. This is reasonable as some F atoms of the TFM groups are more oriented towards the spacer. The stronger F $\cdots H$ interaction in the (TFM) dimer requires the CAAC unit to adopt a more preferable configuration for the hydrogen bonds. This results in the larger dihedral angle, $d_{\mathrm{NCCC}}=20^{\circ}$, in $(\mathrm{CAAC}(\mathrm{TFM}))_{2}$-Ph. The weaker $\mathrm{F} \cdot \mathrm{H}$ interaction allows a smaller $d_{\mathrm{NCCC}}=7^{\circ}$ in the $(\mathrm{F})$ dimer, which favors $\pi$ bonding interaction between the CAAC units and the spacer. The larger dihedral angle in $(\mathrm{CAAC}(\mathrm{TFM}))_{2}-\mathrm{Ph}$ results in less overlap between the carbene $\pi$ orbitals and the LUMO and HOMO of the spacer, and thus the higher diradical character of $(\mathrm{CAAC}(\mathrm{TFM}))_{2}-\mathrm{Ph}$.
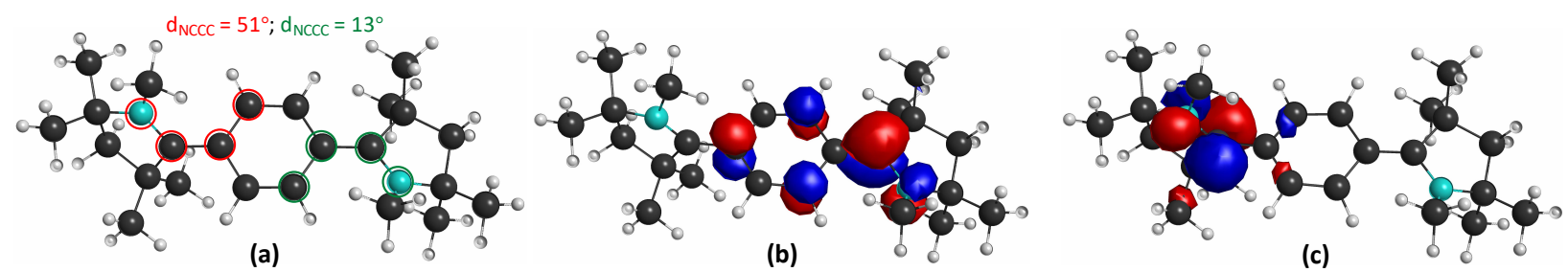

FIG. 8. A representative structure (a) obtained during the optimization of the $S_{1}$ structure of $(\mathrm{CAAC})_{2}-\mathrm{Ph}$ and its (b) HOMO and (c) LUMO.

The 0.43 and $0.62 \mathrm{eV} \Delta E_{S F}^{(1)} \mathrm{s}$ of $(\mathrm{CAAC}(\mathrm{TFM}))_{2}-\mathrm{Ph}$ and $(\mathrm{CAAC}(\mathrm{F}))_{2}-\mathrm{Ph}$ seem large and imply energy waste and slow SF. However, they are not large when compared with the $0.58 \mathrm{eV} \Delta E_{S F}^{(1)}$ of molecular pentacene. The $\Delta E_{S F}^{(1)}$ of this most intensely studied SF chromophore decreases to $0.11 \mathrm{eV}$ in solid state, due to the significant reduction of its adiabatic $E\left(S_{1}\right)$ from 2.31 to $1.86 \mathrm{eV}$. This red-shift of $E\left(S_{1}\right)$ in changing from molecules to their crystals is common for organic molecules and is attributed to the Coulomb interaction between neighboring molecules and Davydov splitting. ${ }^{79}$ The seemingly large $\Delta E_{S F}^{(1)} \mathrm{s}$ of the two dimeric molecules have reserved some room for the red-shifts, so that the dimers in solids can still satisfy Eq. 1. Without running solid state calculations, we cannot predict the red-shifts. However, we note that $E\left(S_{1}\right) \approx 2 E\left(T_{1}\right)$ may not be an essential requirement for isolated molecules in designing SF chromophores.

The unsubstituted dimer $(\mathrm{CAAC})_{2}-\mathrm{Ph}$, if all its structures of the four relevant states maintained $C_{2}$ symmetry, would also be a possible candidate for SF chromophore. The energies in Table III of this dimer are obtained using the $C_{2}$ structures. These energies 
give $\Delta E_{S F}^{(1)}=-0.03 \mathrm{eV}, \Delta E_{S F}^{(2)}=0.20 \mathrm{eV}$, and a $0.14 \mathrm{eV}$ SF barrier. The non-ZPEcorrected vertical $E\left(S_{1}\right)$ and vertical $E\left(T_{1}\right)$ are calculated to be 2.76 and $1.72 \mathrm{eV}$, which are in good agreement with the 2.74 and $1.70 \mathrm{eV}$ calculated values reported in Ref. 44 . The small negative $\Delta E_{S F}^{(1)}$ can be overcome by thermal activation and the barrier is surmountable. However, the $S_{1}$ structure optimized with $C_{2}$ symmetry features an imaginary frequency with a symmetry-breaking mode. The optimization following this mode leads to structure with one CAAC unit being significantly non-coplanar with the spacer. One such representative structure is shown in Figure $8(\mathrm{a})$, with the increased $d_{\mathrm{NCCC}}$ angle marked in red color and the other in green color. The HOMO and LUMO of this structure are shown in Figure 8(b) and $(\mathrm{c})$.

At this structure, the $S_{1}$ state corresponds to an open-shell singlet with one electron in each of the HOMO and LUMO, while the $S_{0}$ state corresponds to double occupation of the HOMO. The $S_{0}-S_{1}$ gap is only $0.24 \mathrm{eV}$. The two states are about to exchange their characters, and they do a few optimization steps later. The $S_{1}$ structural optimization thus failed. Taking $E\left(S_{0}\right) @ S_{0}$ g as the reference, the non-ZPE-corrected $E\left(S_{1}\right)$ at this structure is calculated to be $2.18 \mathrm{eV}$, which is substantially less than $2 E\left(T_{1}\right)=2 \times 1.35=2.70 \mathrm{eV}$. Please note that this $E\left(T_{1}\right)$ does not contain ZPE correction. It is comparable with the non-ZPEcorrected $E\left(S_{1}\right)$. Therefore, even if we can locate the minimum on the $S_{1}$ potential energy surface, $(\mathrm{CAAC})_{2}-\mathrm{Ph}$ would not satisfy Eq. 1 . The $S_{0}-S_{1}$ root flipping in the structural optimization implies that the $S_{1}$ would rapidly decay to the $S_{0}$ state radiationlessly. The short-lived $S_{1}$ also disfavors SF.

The reorganization of the $S_{1}$ structure into this asymmetric configuration can be rationalized as follows. As mentioned above, the singlet open-shell with one electron in each of the delocalized HOMO and LUMO in Figure 5(d) and (e) correspond to a charge resonance closed-shell state, with two electrons in one of the localized orbitals in Figure 6 and none in the other. Such a state features long distance charge transfer between the two CAAC $\pi$ orbitals (e.g., the first resonance structure in Figure 5(i)), which is energy costly. By rotating one CAAC unit out of the plane of the phenylene spacer, the $\pi$ overlap is lost and the delocalized HOMO and LUMO have evolved to the less delocalized HOMO and the localized LUMO in Figure 8(b) and (c). The singlet open-shell with one electron in each of these resultant orbitals does not involve the long distance charge transfer between the CAAC units. The energy of the $S_{1}$ state is hence lower. This tendency to lower the $S_{1}$ 
energy by rotating one CAAC unit out of the plane of the spacer must also be applicable in $\left(\mathrm{CAAC}(\mathrm{TFM})_{2}-\mathrm{Ph}\right.$ and $(\mathrm{CAAC}(\mathrm{F}))_{2}-\mathrm{Ph}$. However, the $\mathrm{F} \cdots \mathrm{H}$ bonds shown in Figure 7 prevent this distortion from happening.

\section{CONCLUSIONS}

With the objective to design new singlet fission chromophores, we employ MRSF-TDDFT and NEVPT2 methods to investigate a series of cyclic (alkyl)(amino)carbenes (CAAC) dimers. In the dimers, the CAAC units are connected by $\mathrm{C}_{2}, \mathrm{C}_{4}$, and $p$-phenylene spacers, and they are substituted by trifluoromethyls and fluorine atoms at their $\alpha \mathrm{C}$ position. The trifluoromethylation and fluorination are found to stabilize the $\pi$ LUMO of CAAC, consequently enhance the diradical character for the CAAC dimers, and make them closer to satisfy or satisfy the $E\left(S_{1}\right) \geq 2 E\left(T_{1}\right)$ primary energy condition for singlet fission. Meanwhile, the $\alpha \mathrm{C}$ remains being a quaternary $\mathrm{C}$. The two substitution schemes will find more use beyond the context of singlet fission, as they can functionalize CAAC in other fields of optoelectronics. The spacers mainly have two effects: (1) the frontier orbitals of a longer spacer $\left(\mathrm{C}_{4}\right.$ vs. $\left.\mathrm{C}_{2}\right)$ are more attenuated and have smaller overlaps with the $\pi$ orbital of the CAAC units, leading to higher diradical characters of the dimers; (2) cylindrically symmetric spacers $\mathrm{C}_{2}$ and $\mathrm{C}_{4}$ allow significant structural reorganizations in the excited states, which potentially lead to endoergicity and high barrier for singlet fission. With both the attenuated frontier orbitals and non-cylindrical symmetry of the spacer, $p$-phenylene-connected dimers with $\mathrm{CF}_{3}$ and $\mathrm{F}$ at the $\alpha \mathrm{C}$ position are found to be promising candidates for both thermodynamically and kinetically favorable singlet fission. The presence of the F atoms results in hydrogen bonds between the CAAC units and the spacer. The hydrogen bonds prevent a $\mathrm{SF}$-unfavorable structural distortion in the $S_{1}$ state, which occurs in the (CAAC $)_{2}$-Ph dimer.

Other than proposing the two chromophore candidates, this study demonstrates the importance of considering structural reorganizations in designing singlet fission chromophores. Considering vertical $E\left(S_{1}\right) \mathrm{s}$ and adiabatic $E\left(T_{1}\right) \mathrm{s}$, all three $\mathrm{C}_{2}$-connected dimers satisfy $E\left(S_{1}\right) \geq 2 E\left(T_{1}\right)$. The significant $S_{1}$ structural reorganizations reverse the signs of $E\left(S_{1}\right)-$ $2 E\left(T_{1}\right)$. The structural reorganizations of the $S_{1}$ and $T_{1}$ states also lead to high barriers for the singlet fission of the $\mathrm{C}_{4}$-connected trifluoromethylated and the fluorinated dimers. The two dimers, although they satisfy (or close to satisfy) $E\left(S_{1}\right) \geq 2 E\left(T_{1}\right)$, are hence not good 
candidates for singlet fission chromophores. It is a common practice to only consider vertical excitation energies in a quick survey of a large amount of structures in designing singlet fission chromophores. However, it is necessary to consider structural reorganizations of the excited states in order to more conclusively judge whether a molecule is a good candidate.

\section{SUPPLEMENTARY MATERIAL}

$\mathrm{HOMO}-1$ of $(\mathrm{CAAC})_{2}-\mathrm{C}_{4}$; coordinates of all studied structures.

\section{ACKNOWLEDGEMENTS}

We thank the Gordon group at the Iowa State University for their continuing development of the GAMESS-US program package. We thank the Neese group at the University of Bonn for their continuing development of the ORCA program package. Computations were made on the supercomputer Mammoth parallelèl 2 from Université de Sherbrooke, managed by Calcul Québec and Compute Canada. T.Z. is thankful to the Natural Sciences and Engineering Research Council (NSERC) of Canada for research funding (RGPIN-201606276), and Carleton University for start-up grant (186853). S.L. and C.H.C. acknowledge the support by the Samsung Science and Technology Foundations (SSTF-BA1701-12). 
1 M. B. Smith and J. Michl, Chem. Rev. 110, 6891 (2010).

2 M. B. Smith and J. Michl, Annu. Rev. Phys. Chem. 64, 361 (2013).

3 W. Shockley and H. J. Queisser, J. Appl. Phys. 32, 510 (1961).

4 M. C. Hanna and A. J. Nozik, J. Appl. Phys. 100, 074510 (2006).

5 K. C. Krishnapriya, A. J. Musser, and S. Patil, ACS Energy Lett. 4, 192 (2019).

6 D. Casanova, Chem. Rev. 118, 7164 (2018).

7 W.-L. Chan, M. Ligges, and X.-Y. Zhu, Nature Chem. 4, 840 (2012).

8 W.-L. Chan, M. Ligges, A. Jailaubekov, L. Kaake, L. Miaja-Avila, and X.-Y. Zhu, Science 334, 1541 (2011).

9 D. N. Congreve, J. Lee, N. J. Thompson, E. Hontz, S. R. Yost, P. D. Reusswig, M. E. Bahlke, S. Reineke, T. Van Voorhis, and M. A. Baldo, Science 340, 334 (2013).

10 T. Zeng, R. Hoffmann, and N. Ananth, J. Am. Chem. Soc. 136, 5755 (2014).

11 S. R. Yost, J. Lee, M. W. B. Wilson, T. Wu, D. P. McMahon, R. R. Parkhurst, N. J. Thompson, D. N. Congreve, A. Rao, K. Johnson, M. Y. Sfeir, M. Bawendi, T. M. Swager, R. H. Friend, M. A. Baldo, and T. Van Voorhis, Nature Chem. 6, 492 (2014).

12 R. D. Pensack, A. J. Tilley, S. R. Parkin, T. S. Lee, M. M. Payne, D. Gao, A. A. Jahnke, D. G. Oblinsky, P.-F. Li, J. E. Anthony, D. S. Seferos, and G. D. Scholes, J. Am. Chem. Soc. 137, $6790(2015)$.

13 B. J. Walker, A. J. Musser, D. Beljonne, and R. H. Friend, Nature Chem. 5, 1019 (2013).

14 D. Casanova, J. Chem. Theory Comput. 10, 324 (2014).

15 E. A. Margulies, Y.-L. Wu, P. Gawel, S. A. Miller, L. E. Shoer, R. D. Schaller, F. Diederich, and M. R. Wasielewski, Angew. Chem. Int. Ed. 54, 8679 (2015).

16 S. N. Sanders, E. Kumarasamy, A. B. Pun, M. T. Trinh, B. Choi, J. Xia, E. J. Taffet, J. Z. Low, J. R. Miller, X. Roy, X.-Y. Zhu, M. L. Steigerwald, M. Y. Sfeir, and L. M. Campos, J. Am. Chem. Soc. 137, 8965 (2015).

17 E. G. Fuemmeler, S. N. Sanders, A. B. Pun, E. Kumarasamy, T. Zeng, K. Miyata, M. L. Steigerwald, X.-Y. Zhu, M. Y. Sfeir, L. M. Campos, and N. Ananth, ACS Cent. Sci. 2, 316 (2016).

18 S. N. Sanders, E. Kumarasamy, A. B. Pun, K. Appavoo, M. L. Steigerwald, L. M. Campos, 
and M. Y. Sfeir, J. Am. Chem. Soc. 138, 7289 (2016).

19 N. H. Damrauer and J. L. Snyder, J. Phys. Chem. Lett. 6, 4456 (2015).

20 J. D. Cook, T. J. Carey, and N. H. Damrauer, J. Phys. Chem. A 120, 4473 (2016).

21 Y. Wu, K. Liu, H. Liu, Y. Zhang, H. Zhang, J. Yao, and H. Fu, J. Phys. Chem. Lett. 5, 3451 (2014).

22 S. W. Eaton, L. E. Shoer, S. D. Karlen, S. M. Dyar, E. A. Margulies, B. S. Veldkamp, C. Ramanan, D. A. Hartzler, S. Savikhin, T. J. Marks, and M. R. Wasielewski, J. Am. Chem. Soc. 135, 14701 (2013).

23 E. A. Margulies, C. E. Miller, Y. Wu, L. Ma, G. C. Schatz, R. M. Young, and M. R. Wasielewski, Nature Chem. 8, 1120 (2016).

24 H.-H. Lin, K. Y. Kue, G. C. Claudio, and C.-P. Hsu, J. Chem. Theory Comput. 15, 2246 (2019).

25 C. Wang and M. J. Tauber, J. Am. Chem. Soc. 132, 13988 (2010).

26 R. J. Dillon, G. B. Piland, and C. J. Bardeen, J. Am. Chem. Soc. 135, 17278 (2013).

27 A. J. Musser, M. Maiuri, D. Brida, G. Cerullo, R. H. Friend, and J. Clark, J. Am. Chem. Soc. 137, $5130(2015)$.

28 J. Xia, S. N. Sanders, W. Cheng, J. Z. Low, J. Liu, L. M. Campos, and T. Sun, Adv. Mater. 29, $1601652(2017)$.

29 I. Paci, J. C. Johnson, X. D. Chen, G. Rana, D. Popović, D. E. David, A. J. Nozik, M. A. Ratner, and J. Michl, J. Am. Chem. Soc. 128, 16546 (2006).

30 T. Minami and M. Nakano, J. Phys. Chem. Lett. 3, 145 (2012).

31 T. Minami, S. Ito, and M. Nakano, J. Phys. Chem. Lett. 4, 2133 (2013).

32 S. Ito, T. Nagami, and M. Nakano, J. Photochem. Photobiol. 34, 85 (2018).

33 A. Japahuge and T. Zeng, ChemPlusChem 83, 146 (2018).

34 A. F. Schwerin, J. C. Johnson, M. B. Smith, P. Sreearunothai, D. Popović, J. Černý, Z. Havlas, I. Paci, A. Akdag, M. K. MacLeod, X. Chen, D. E. David, M. A. Ratner, J. R. Miller, A. J. Nozik, and J. Michl, J. Phys. Chem. A 114, 1457 (2010).

35 J. N. Schrauben, J. L. Ryerson, J. Michl, and J. C. Johnson, J. Am. Chem. Soc. 136, 7363 (2014).

36 J. C. Johnson and J. Michl, Top. Curr. Chem. 375, 80 (2017).

37 A. Akdag, Z. Havlas, and J. Michl, J. Am. Chem. Soc. 134, 14624 (2012). 
38 J. Wen, Z. Havlas, and J. Michl, J. Am. Chem. Soc. 137, 165 (2015).

39 T. Zeng, N. Ananth, and R. Hoffmann, J. Am. Chem. Soc. 136, 12638 (2014).

40 T. Zeng and P. Goel, J. Phys. Chem. Lett. 7, 1351 (2016).

41 T. Zeng, J. Phys. Chem. Lett. 7, 4405 (2016).

42 T. Zeng, S. K. Mellerup, D. Yang, X. Wang, S. Wang, and K. Stamplecoskie, J. Phys. Chem. Lett. 9, 2919 (2018).

43 K. Bhattacharyya, S. M. Pratik, and A. Datta, J. Phys. Chem. C 119, 25696 (2015).

44 J. Messelberger, A. Grünwald, P. Pinter, M. M. Hansmann, and D. Munz, Chem. Sci. 9, 6107 (2018).

45 M. Soleilhavoup and G. Bertrand, Acc. Chem. Res. 48, 256 (2015).

46 M. M. Hansmann, M. Melaimi, D. Munz, and G. Bertrand, J. Am. Chem. Soc. 140, 2546 (2018).

47 R. Kinjo, B. Donnadieu, and G. Bertrand, Angew. Chem. Int. Ed. 49, 5930 (2010).

48 O. Back, B. Donnadieu, P. Parameswaran, G. Frenking, and G. Bertrand, Nature Chem. 2, $369(2010)$

49 O. Back, M. A. Celik, G. Frenking, M. Melaimi, B. Donnadieu, and G. Bertrand, J. Am. Chem. Soc. 132, $10262(2010)$.

50 R. Kinjo, B. Donnadieu, M. A. Celik, G. Frenking, and G. Bertrand, Science 333, 610 (2011).

51 V. Lavallo, Y. Canac, B. Donnadieu, W. W. Schoeller, and G. Bertrand, Angew. Chem. Int. Ed. 46, 3488 (2006).

52 H. G. Viehe, Z. Janousek, R. Merenyi, and L. Stella, Acc. Chem. Res. 18, 148 (1985).

53 D. J. Pasto, J. Am. Chem. Soc. 110, 8164 (1988).

54 S. Lee, M. Filatov, S. Lee, and C. H. Choi, J. Chem. Phys. 149, 104101 (2018).

55 S. Lee, E. E. Kim, H. Nakata, S. Lee, and C. H. Choi, J. Chem. Phys. 150, 184111 (2019).

56 C. Lee, W. Yang, and R. G. Parr, Phys. Rev. B 37, 785 (1988).

57 A. D. Becke, J. Chem. Phys. 98, 5648 (1993).

58 F. .Weigend and R. Ahlrichs, Phys. Chem. Chem. Phys. 7, 3297 (2005).

59 M. W. Schmidt, K. K. Baldridge, J. A. Boatz, S. T. Elbert, M. S. Gordon, J. H. Jensen, S. Koseki, N. Matsunaga, K. A. Nguyen, S. Su, T. L. Windus, M. Dupuis, and J. A. Montgomery Jr., J. Comput. Chem. 14, 1347 (1993).

60 M. S. Gordon and M. W. Schmidt, "Advances in electronic structure theory: Gamess a decade 
later," in Theory and Applications of Computational Chemistry: The First Forty Years, edited by C. E. Dykstra, G. Frenking, K. S. Kim, and G. E. Scuseria (Elsevier, Amsterdam, 2005) Chap. 41, pp. 1167-1189.

61 J.-L. Brédas, D. Beljonne, V. Coropceanu, and J. Cornil, Chem. Rev. 104, 4971 (2004).

62 V. May and O. Kühn, Charge and Energy Transfer Dynamics in Molecular Systems., 3rd ed. (WILEY-VCH, Weinheim, 2011).

63 H. Geng, Y. Niu, Q. Peng, Z. Shuai, V. Coropceanu, and J.-L. Brédas, J. Chem. Phys. 135, 104703 (2011).

64 C. Angeli, R. Cimiraglia, S. Evangelisti, T. Leininger, and J.-P. Malrieu, J. Chem. Phys. 114, $10252(2001)$.

65 C. Angeli, R. Cimiraglia, and J.-P. Malrieu, Chem. Phys. Lett. 328, 297 (2001).

66 C. Angeli, R. Cimiraglia, and J.-P. Malrieu, J. Chem. Phys. 117, 9138 (2002).

67 F. Neese, WIRES Comput. Mol. Sci. 2, 73 (2012).

68 I. Mayer, Int. J. Quantum Chem. 26, 151 (1984).

69 R. Hoffmann, Acc. Chem. Res. 4, 1 (1971).

70 W. P. Ozimiński and J. C. Dobrowolski, J. Phys. Org. Chem. 22, 769 (2009).

71 M. Rahm, T. Zeng, and R. Hoffmann, J. Am. Chem. Soc. 141, 342 (2019).

72 J. E. True, D. Thomas, R. W. Winter, and G. L. Gard, Inorg. Chem. 42, 4437 (2003).

73 L. Jin, M. Melaimi, L. Liu, and G. Bertrand, Org. Chem. Front. 1, 351 (2014).

74 Y. Li, K. C. Mondal, P. P. Samuel, H. Zhu, C. M. Orben, S. Panneerselvam, B. Dittrich, B. Schwederski, W. Kaim, T. Mondal, D. Koley, and H. W. Roesky, Angew. Chem. Int. Ed. 53, 4168 (2014).

75 C. R. Flynn and J. Michl, J. Am. Chem. Soc. 96, 3280 (1974).

76 R. A. Marcus, Rev. Mod. Phys. 65, 599 (1993).

77 S. Shaik and P. C. Hiberty, A chemist's guide to valence bond theory (Wiley-Interscience, New Jersey, 2008).

78 G. K. S. Prakash, F. Wang, M. Rahm, J. Shen, C. Ni, R. Haiges, and G. A. Olah, Angew. Chem. Int. Ed. 50, 11761 (2011).

79 M. Schwoerer and H. C. Wolf, Organic Molecular Solids (WILEY-VCH Verlag GmbH and Co. KGaA, Weinheim, 2007). 